\title{
Visibilidade em Coloproctologia está Correlacionada com Atividade em Pesquisa
}

\author{
Visibility in Colorrectal Surgery is Related with Research Activity
}

\author{
ANTONIO SÉRGIO BRENNER ${ }^{1}$; VANESSAZENIDE LIMA ${ }^{2}$; MICHELE VARASCHIM ${ }^{2}$; PAOLAZARUR VARELLA $^{2}$; \\ BRUNOZENIDELIMA ${ }^{3}$; SÉRGIOBRENNER $^{4}$
}

\author{
${ }^{1}$ Professor Adjunto e Médico do Serviço de Coloproctologia do Hospital Universitário Evangélico \\ de Curitiba; ${ }^{2}$ Residente do Serviço de Coloproctologia do Hospital Universitário Evangélico de Curitiba; \\ ${ }^{3}$ Residente do Serviço de Cirurgia Geral do Hospital Universitário Evangélico de Curitiba; ${ }^{4}$ Professor Adjunto e \\ Chefe do Serviço de Cirurgia Geral do Hospital Evangélico de Curitiba - Paraná - Brasil.
}

\begin{abstract}
BRENNER AS; LIMA VZ; VARASCHIM M; VARELLA PZ; LIMA BZ; BRENNER S. Visibilidade em Coloproctologia está Correlacionada com Atividade em Pesquisa. Rev bras Coloproct, 2008;28(3): 319-323.

RESUMO: Introdução: $O$ objetivo do presente estudo foi avaliar a influencia da atividade médica em pesquisa, com a exposição que o coloproctologista tem nos congressos da Sociedade Brasileira de Coloproctologia (SBCP). Métodos: Comparamos o número de publicações em revistas indexadas ao Medline e Lilacs de cada palestrante dos últimos cinco congressos da SBCP, com o número de publicações de médicos sócios não palestrantes, selecionados aleatoriamente. A pesquisa incluiu o ano de 1965 até 2005, respeitando o mesmo tempo de formado e estado de atuação profissional. Resultados: Foram selecionados um total de 13 conferencistas e 88 palestrantes dos últimos 5 congressos da SBCP, que foram comparados com 102 médicos sócios não palestrantes da SBCP. Os palestrantes publicaram mais trabalhos científicos que os não palestrantes $(\mathbf{p}<0,0009)$, e os conferencistas produzi-

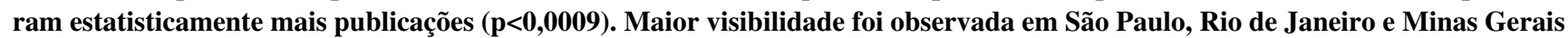
$(71,8 \%, 6,5 \%$ e $6,5 \%$ dos palestrantes respectivamente), e maior atividade científica foi observada entre 20 e 40 anos de atividade profissional. A grande maioria das publicações foi em revistas nacionais, especialmente na revista da SBCP (aproximadamente 45\%). Conclusão: A exposição do médico como convidado nos congressos da SBCP e o volume de produção científica estão correlacionados.
\end{abstract}

Descritores: Relações interpessoais; liderança; visibilidade.

\section{INTRODUÇÃO}

O destaque que o médico alcança durante sua carreira profissional, é resultado de sua atuação clínica, suas realizações e posição profissional que ocupa. Assim como ocorre em qualquer atividade, a posição, remuneração e o reconhecimento entre seus pares, é fruto de trabalho intenso e do talento natural de cada profissional $(1,2)$.

O sucesso profissional tem como conseqüência o inevitável convite para eventos, entrevistas e matérias em meio jornalístico leigo. Através da intensi- dade e qualidade da produção científica, seria possível diferenciar o profissional de destaque, entre os convidados apenas bem relacionados e com conhecimento teórico insuficiente ou produção profissional irrelevante. A atividade em pesquisa se traduz principalmente pelo número de publicações, fator de impacto da revista e pelo número de citações que o autor recebeu(3). Por outro lado, a produtividade científica é apenas um aspecto dos desafios que a evolução da medicina experimenta. Médicos com grande experiência prática não devem ser desqualificados, e tem uma grande contribuição a oferecer.

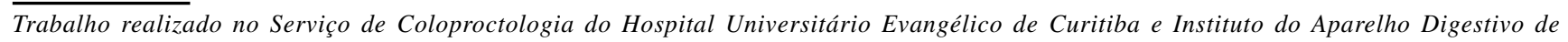
Curitiba. Curitiba-PR - Brasil. 
Entre os médicos, ser convidado como palestrante em congresso é também receber destaque especial. Em congressos médicos, existe um sentimento velado de que alguns dos palestrantes seriam convidados por relacionamento pessoal ou indicação de conveniência política. $\mathrm{O}$ palestrante ganha visibilidade e até forma opinião entre seus colegas. Nos casos de grande envolvimento, torna-se referência com repercussões em seu consultório particular.

O objetivo do presente estudo foi avaliar a relação da atividade médica em pesquisa, com a exposição que o coloproctologista tem nos congressos da Sociedade Brasileira de Coloproctologia (SBCP).

\section{MÉTODOS}

Entendemos como visibilidade a exposição do médico aos seus colegas, ao ser convidado como palestrante em eventos médicos.

Analisamos os programas científicos dos últimos 5 Congressos Brasileiros de Coloproctologia, e selecionamos os palestrantes brasileiros convidados para atuarem como conferencistas ou palestrantes nas sessões de mesa redonda nos últimos cinco congressos da SBCP. Excluímos o congresso realizado em 2007. Ano em que apresentamos esse trabalho, e teve um dos autores em sua comissão organizadora. Após essa etapa, selecionamos aleatoriamente e por pareamento, outros membros (associados ou titulares) da sociedade, mas não palestrantes. $\mathrm{Na}$ alocação dos grupos, respeitamos uma proporcionalidade por estado e por tempo de formatura.

Foram pesquisados os artigos publicados em revistas indexadas ao Medline e Lilacs por todos os médicos selecionados, entre os anos de 1965 até 2005. Avaliamos a atividade em pesquisa através da contagem simples do número de publicações nas revistas indexadas para cada profissional. O número de publicações dos palestrantes (conferencistas e participantes em mesa redonda) foi comparado com o número de publicações de médicos também sócios da SBCP, mas não-palestrantes.

Utilizamos o teste estatístico t de "student" para comparação dos grupos adotando-se nível de significância para $\mathrm{p}<0,05$.

\section{RESULTADOS}

Foram selecionados um total de 13 conferencistas e 88 palestrantes dos últimos 5 congressos da
SBCP, que foram comparados com 102 médicos sócios da SBCP mas não palestrantes.

Os 13 conferencistas publicaram um total de 325 trabalhos (média de 25 artigos cada), e os 88 palestrantes em sessões de mesa redonda, 722 trabalhos (média de 8,2 artigos cada). O grupo comparativo de 102 médicos não-palestrantes publicaram um total de 235 trabalhos (média de 2,3 artigos cada).

Os médicos palestrantes (conferencistas + palestrantes de mesa redonda) publicaram estatisticamente mais trabalhos científicos que os sócios da SBCP não palestrantes - média de 11,62 publicações para cada palestrante e 2,36 por membros da SBCP não palestrantes $(\mathrm{p}<0,0009)$. Os palestrantes conferencistas, quando isoladamente avaliados, produziram estatisticamente mais publicações quando comparados ao grupo de palestrantes de mesas redondas - média de 25 X 8,2 publicações $(\mathrm{p}<0,0009)$.

Maior atividade científica foi observada em SP, e nos médicos entre 20 e 40 anos de formado. A maior visibilidade (convite como palestrante) em congressos foi dos paulistas com $71,8 \%$, seguido pelos palestrantes dos estados do Rio de Janeiro e Minas Gerais com 6,5\% cada. O estado de São Paulo obteve também a maior média de trabalhos publicados por profissional com 12,8 publicações, seguido por Santa Catarina (10 publicações), Paraná (6,7 publicações) e Ceará (6,6 publicações). (Tabela 1)

A grande maioria das publicações foi em revistas nacionais, especialmente na revista da SBCP tanto nos palestrantes (com 37,19\%), como nos não palestrantes $(52,7 \%)$, seguida pela revista do Colégio

\section{Tabela 1}

\begin{tabular}{lcc}
\hline Estado & $\begin{array}{c}\text { Visibilidade } \\
\text { Média }\end{array}$ & Publicações \\
\hline São Paulo & $71,8 \%$ & 12,8 \\
Minas Gerais & $6,5 \%$ & 2,1 \\
Rio de Janeiro & $6,5 \%$ & 1,79 \\
Paraná & $5,68 \%$ & 6,7 \\
Ceará & $4,5 \%$ & 6,6 \\
Bahia & $4,0 \%$ & 3,3 \\
Goiás & $2,2 \%$ & 4,3 \\
Santa Catarina & $1,78 \%$ & 10 \\
Distrito Federal & $1,35 \%$ & 4 \\
Rio Grande do Sul & $0,8 \%$ & 1,25 \\
Pernambuco & $0,17 \%$ & 0,33 \\
\hline
\end{tabular}


Brasileiro de Cirurgiões com 6,21\% e 10,37\% nos grupos palestrantes e não palestrantes, respectivamente. (Gráficos 1 e 2)

\section{DISCUSSÃO}

Um melhor desempenho, mesmo que discreto, confere ao vencedor todos os créditos e benesses(4). O profissional de sucesso transmite benefícios simultâneos(5) e no caso dos médicos, os beneficiários seriam eles mesmos (de forma pessoal), os pacientes em geral e os médicos que com maior conhecimento adquirido, evoluem em sua carreira. No Brasil, a pesqui- sa pouco atrai os médicos envolvidos exclusivamente em assistência. O hábito de manter um banco de dados, publicar seus resultados e discuti-los com a literatura, mantém o cirurgião atualizado e o diferencia, direcionando sua prática à uma área específica. Com o maior conhecimento adquirido e com interesse focado em um assunto particular, o médico tem o potencial de tornar-se um expert com condições de propor refinamentos técnicos ou novos tratamentos.

Número de publicações, citações recebidas pelo autor e fator de impacto da revista em que o trabalho foi publicado trazem uma boa noção da intensidade e qualidade da produção científica do autor. Traba-

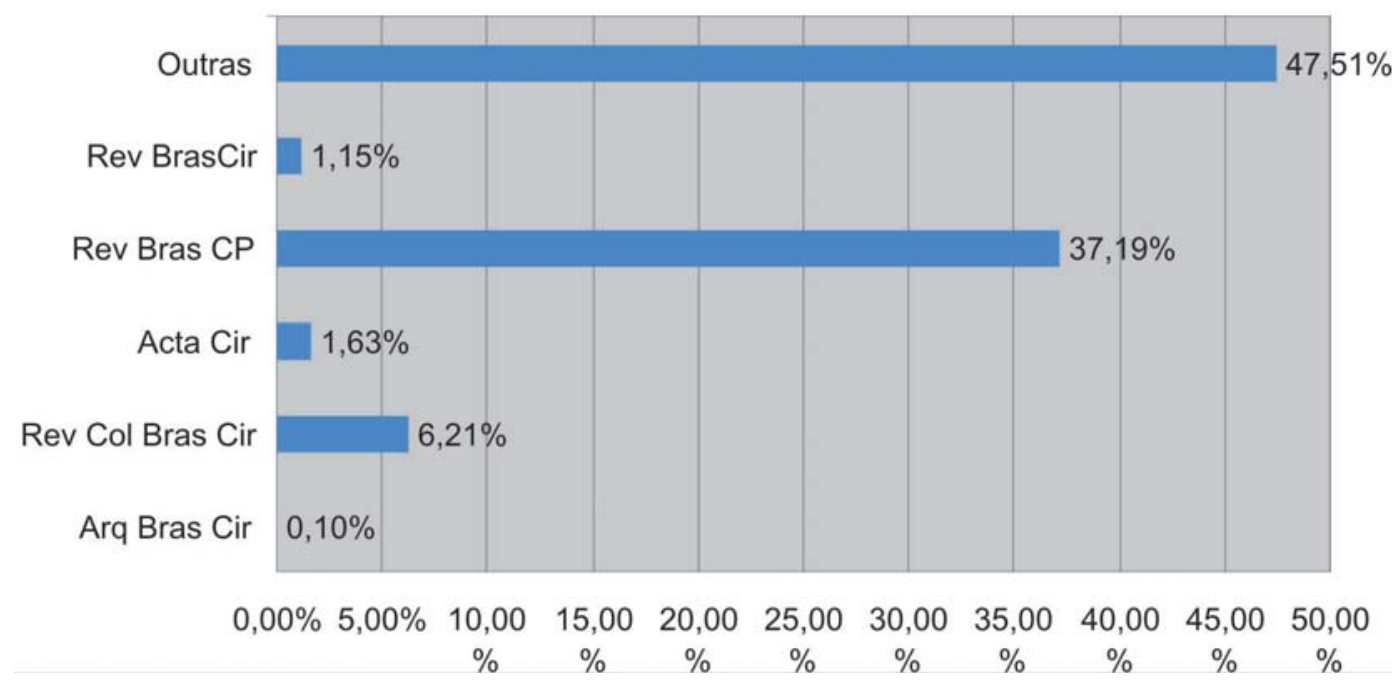

Gráfico 1 - Publicações em revistas dos palestrantes.

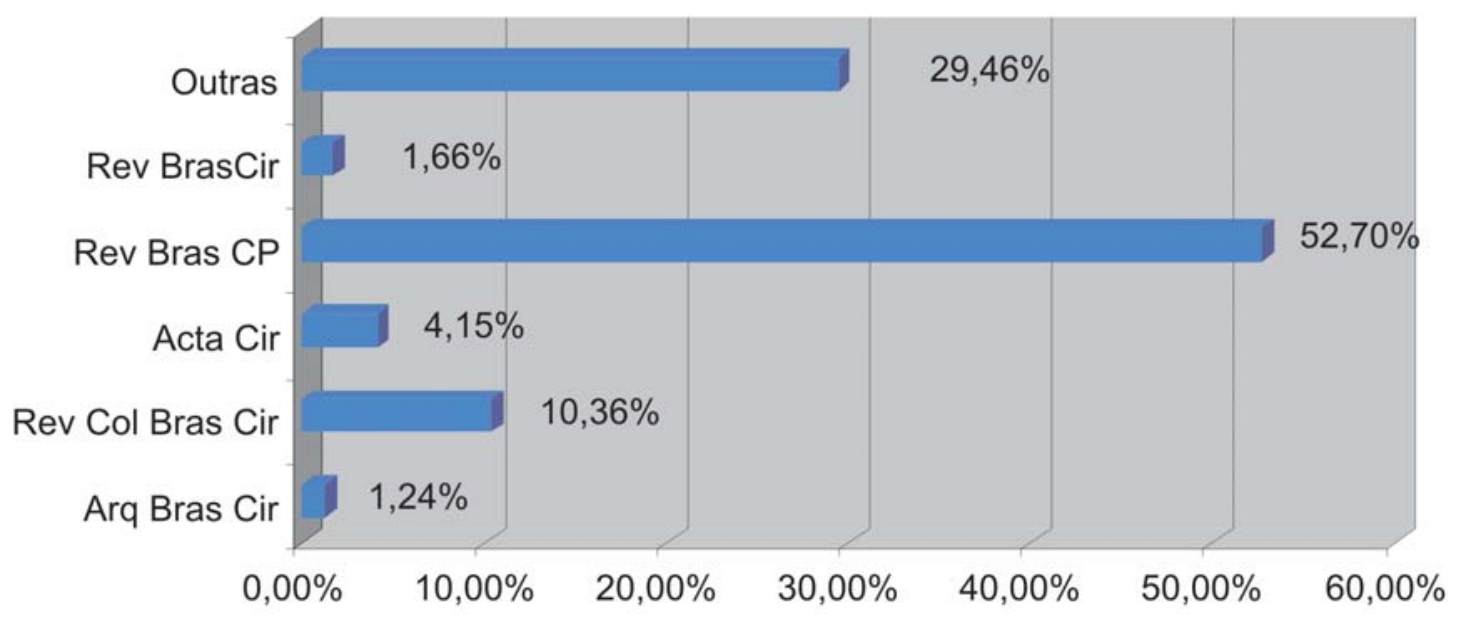

Gráfico 2 - Publicações em revistas dos não-palestrantes. 
lhos de visibilidade internacional (listadas no Science Citation Index database) ou local da publicação (publicações em língua nativa de revistas não indexadas), somam-se ao número de citações recebidas e fator de impacto da revista, e podem ser facilmente acessados na internet. Esses dados são usualmente utilizados em seleção profissional, para financiamento de pesquisa, e estão relacionados à exposição do profissional (3).

A visibilidade que forma opiniões sofreu grande interferência recente. Com a informática e disponibilidade da internet na mesa do consultório, o médico pode acessar artigos, consensos ou opiniões de diversos autores. Murali e cols. (2004) demonstraram que os artigos com resumo ou texto completo disponíveis na Internet, aumentam o fator de impacto da revista. Não é só no congresso que o médico tem a possibilidade de aumentar sua visibilidade e formar opinião(6). A web tem um poder ainda a ser adequadamente avaliado.

O estado de São Paulo representa aproximadamente $30 \%$ dos associados à SBCP, mas teve um de seus membros convidado como palestrante em mais de $70 \%$ dos congressos da SBCP. Mesmo considerando-se o maior número de associados, a maior exposição que o cirurgião paulista recebe deve-se ao seu grande desenvolvimento científico e provavelmente, ao ambiente competitivo de suas instituições. Fato esse corroborado pelo maior número de publicações. O dobro do segundo estado que mais publicou no período. Existe portanto, uma relação entre atividade em pesquisa e destaque profissional.

Estratificamos os grupos de cada estado por tempo de formatura dos associados. Não foi possível obter-se a idade de cada profissional. Evitamos assim, comparações entre médicos com tempo de atuações profissionais muito distintas. Um coloproctologista recém saído da sua residência, dificilmente seria um palestrante no congresso da SBCP, e certamente teria menor número de publicações que um médico sênior lotado em um serviço de grande volume de publicações.

Uma tendência observada em congressos recentes é dar exclusividade à convidados internacionais nas sessões de conferência. Os poucos palestrantes nacionais seriam médicos de grande renome e experiência, habitualmente com um volume grande publicações. De uma forma geral, conferencistas nacionais participariam das sessões de mesa redonda ou painel. Por esse motivo, identificamos apenas 13 conferencistas nos últimos 5 anos, contra 88 palestrantes em mesa redonda.

Os nossos resultados demonstram que a Sociedade Brasileira de Coloproctologia busca cada vez mais os profissionais cientificamente qualificados. Essa conduta estimula os novos membros a buscar uma maior qualificação, e dá segurança aos congressistas, que podem confiar na existência de um critério técnico na escolha dos palestrantes.

\section{CONCLUSÃO}

A exposição do médico como convidado nos congressos da SBCP e o volume de produção científica estão correlacionados. Os Serviços credenciados pela SBCP devem incentivar e financiar a pesquisa. A revista da SBCP serve como meio de divulgação adequado pois é onde a maioria dos membros da nossa sociedade apresenta seus resultados.

\footnotetext{
ABSTRACT: Introduction: The aim of the present study was to evaluate the influence of medical research with the exposition that the colorectal surgeon has in the Brazilian colorectal society national congress (SBCP). Methods: We selected all the Brazilian presenters from conferences and round tables sessions of the last 5 national congresses. Presenter's number of publications in journals indexed in Medline and Lilacs were compared to non-presenters surgeons randomly selected. The research included years 1965 to 2005. Groups were selected respecting age and state. Results: Thirteen congress participants and 88 presenters were selected and compared with 102 non-presenters from the SBCP. Presenters published significantly more scientific papers than non-presenters surgeons. More visibility were observed in São Paulo, Rio de Janeiro and Minas Gerais state $(\mathbf{7 1 , 8 \%}, \mathbf{6 , 5 \%}$ and $\mathbf{6 , 5 \%}$ of the presenters respectively), and more research activity was observed in doctors graduated 20 to 40 years ago. The vast majority of publications were printed in national journals, especially in the SBCP journal (near 45\%). Conclusion: The doctor exposition as a presenter in the Brazilian National SBCP Congress and publication volume is correlated.
}

Key words: Interpersonal relations; leadership; visibility. 


\section{REFERÊNCIAS}

1. McConnell CR. Balancing inside and outside: preparing for tomorrow while fulfilling today's responsibilities. Health Care Manag (Frederick). 1999 Dec;18(2):73-82.

2. Strickland B, Arnn J. Orientation on understanding interpersonal influence. J Am Diet Assoc. 1977 Sep;71(3):229-34.

3. Bordons M, Zulueta MA. [Evaluation of the scientific activity through bibliometric indices]. Rev Esp Cardiol. 1999 Oct;52(10):790-800.

4. Reynolds TM, Wierzbicki AS. Does activity in research correlate with visibility? J Clin Pathol. 2004 Apr;57(4):426-7.
5. Friedman SD. Be a better leader, have a richer life. Harv Bus Rev. 2008 Apr;86(4):112-8, 38.

6. Murali NS, Murali HR, Auethavekiat P, Erwin PJ, Mandrekar JN, Manek NJ, et al. Impact of FUTON and NAA bias on visibility of research. Mayo Clin Proc. 2004 Aug;79(8):1001-6.

\section{Endereço para correspondência:}

DR. ANTONIO SÉRGIO BRENNER

Rua de Paz, 156 - Curitiba - PR - Brasil

80060-160

Tel / FAX: 554132634474

E-mail: drbrenner@iadcuritiba.com.br 